\title{
Using semantic memory to find vs create a mood*
}

\author{
ELIZABETH F. LOFTUS \\ University of Washington, Seattle, Washington 98105 \\ SIF WIKSTEN \\ Rutgers University, New Brunswick, New Jersey 08903
}

and

\author{
ROBERT P. ABELSON \\ Yale University, New Haven, Connecticut 06520
}

\begin{abstract}
Ss produced words from semantic memory which satisfied certain constraints. In Experiment I, a noun eategory plus a number and a letter were presented (e.g., ANIMAL-1-D, FRUIT-P-3), and S produced an instance of the category which had the given letter in the position designated by the given number. Faster responses occurred when the position cue occurred before the letter rather than after it. In Experiments II and III, Ss saw only a number and a letter (e.g., 1-D, P-3) and were required to produce any word that had the given letter in the position designated by the given number. Order of the position and letter cues did not influence response times. The effect of order in one case and not the other strongly suggests that producing a word that satisfies certain semantic constraints involves a different process from producing a word that satisfies only certain orthographic restrictions.
\end{abstract}

Given a random English word, is it more likely that the word starts with a $\mathrm{K}$ or that $\mathrm{K}$ is its third letter? Most people judge the first position to be more likely, despite the fact that there are more words in a typical English text in which $\mathrm{K}$ is in the third position (Tversky \& Kahneman, 1972). Why does this happen? Tversky and Kahneman propose that, in making these kinds of frequency judgments, people first attempt to recall some instances of the two classes of words and then base their judgment of frequency on the relative ease with which these instances come to mind. Since "it is certainly easier to think of words that start with a $K$ than of words where $\mathrm{K}$ is in the third position [p. 7]," instances of the former class will be judged more numerous than instances of the latter.

The preceding arguments provided the impetus for the present research. Is it easier to think of words that start with a particular letter than words which have that letter in the third position? Informal testing of ourselves and others suggests that it is. Our initial concern was with two questions: (1) How much easier is it and (2) what is the nature of the retrieval operations that underlie the two cases? Are we dealing with one retrieval strategy or two? Our later concern, however, after examining the results of the first two experiments, was with the difference between "finding" a word in memory and "creating" one. We shall have more to say about this later.

These issues are a subset of the larger problem of how information is organized and retrieved from semantic memory. The present three experiments, all of which

*R equests for reprints should be sent to E. Loftus, Department of Psychology, University of Washington, Seattle, Washington, 98195. The research was supported by Grant MH 22141 to E. Loftus from the National Institute of Mental Health. The assistance of William Cole, Steve Dassin, and Steve Oppenheim is gratefully acknowledged. require $S$ to produce words from semantic memory, provide further data about important aspects of that retrieval.

\section{EXPERIMENT I}

\section{Method}

Subjects. The Ss were 24 students at the New School for Social Research. Each $S$ took part in one experimental session that lasted about $40 \mathrm{~min}$.

Materials. Twenty-five categories were chosen from the Battig and Montague (1969) category norms. Categories that had appeared in previous studies were selected so as to provide some continuity with previous findings. Each category was paired with two restricting cues: (1) a position cue (either the number " 1 " or "3") and (2) a letter cue. The particular pairings of categories with numbers and letters were selected so that a stimulus could refer to the most dominant member of the category (e.g., "animal-1-D" and "animal-3-G" would both allow the response dog, which is the most frequent word given to animal according to the Battig and Montague norms).

The category was always presented first, but the order of the position cue ( 1 or 3 ) and the letter cue was varied; the let ter came either before or after the number, with an interval of $0.5 \mathrm{sec}$. Thus, a particular category could appear in one of four conditions (e.g., "animal-1-D," "animal-D-1," "animal-3-G," "animal-G-3").

Each $S$ received a random permutation of 25 items, with the following restrictions: (1) For a given $\mathrm{S}$, each category appeared only once; (2) in each set of four Ss, a category appeared once in each of the four possible conditions; (3) each $S$ received an approximately equal number of each of the four types of items. In other words, on roughly half of his trials, the position cue " 1 " appeared, while " 3 " appeared on the remaining trials. On roughly half of the trials the position cue came $0.5 \mathrm{sec}$ before the letter; on the remaining trials it came afterward.

Procedure. Each S was told that we were conducting a study on how memory worked, that he would be presented with items consisting of a category, a number, and a letter, and that he was to respond with a word in the category that had the given letter in the position designated by the given number. He was given examples and told to respond as quickly as possible but to avoid errors. 


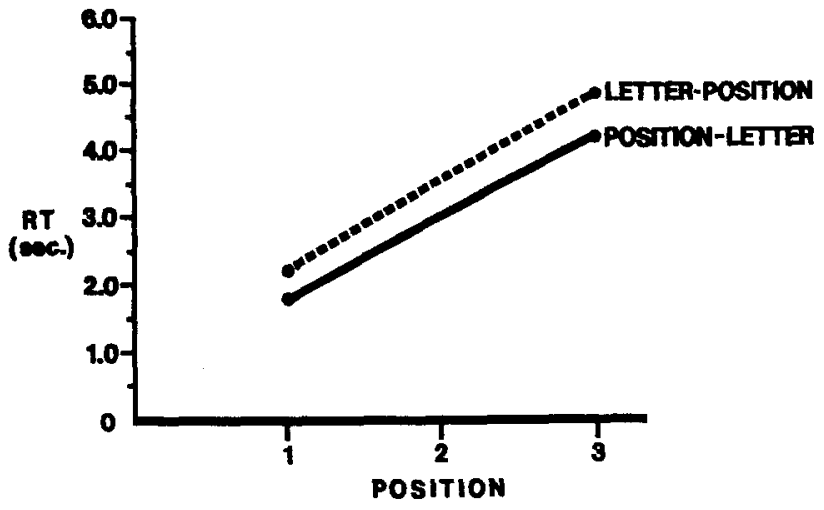

Fig. 1. Results of Experiment I. Mean RT as a function of position cue for position-letter and letter-position orders of presentation.

The $S$ sat in front of a screen in which was a window covered by half-silvered glass. An index card containing the stimulus was placed in a dark enclosure behind the mirror and was presented by illuminating the enclosure. The $S$ responded by speaking into a microphone that was placed in front of him.

A trial consisted of the following: As a card with a letter and a number printed in large type was placed in the darkened enclosure behind the half-silvered mirror, the $E$ spoke the name of the category and pressed a button that illuminated the first cue. After a $0.5-\mathrm{sec}$ interval, the second cue was automatically illuminated and simultaneously an electric timer with a DC clutch was started. The S's verbal response activated a voice key that stopped the clock and terminated the trial. If $S$ did not respond to the stimulus within $30 \mathrm{sec}$, the trial was terminated. A warm-up period of 24 trials preceded the experimental trials.

\section{Results}

Only latencies for correct responses are included in the first two analyses. For each of the four types of stimuli, a mean latency was obtained by averaging medians for individual Ss. These means are presented in Fig. 1 .

A two-way analysis of variance of the latencies in terms of (1) position cue (1 vs 3 ) and (2) order of position and letter cues (e.g., 1-D vs D-1) revealed that Ss produced a category member more quickly when provided with the first letter than when provided with the third letter $[\mathrm{F}(1,23)=62.54, \mathrm{p}<.01]$ and more quickly when given the position cue before the letter cue rather than afterward $[F(1,23)=5.99, p<.05]$. The interaction was not significant $(F<1)$. The least significant difference test (Kirk, 1968) for testing for differences between all possible pairs of means led to all six pairwise comparisons reaching at least the .05 level of significance.

A similar analysis was done by categories; that is, for each of the four types of stimuli, a mean latency was obtained by averaging medians for individual categories. A two-way analysis of variance of the latencies in terms of position cue and order of position and letter cues revealed that $S s$ produced a category member more quickly when provided with the first letter than when provided with the third letter $[\mathrm{F}(1,24)=96.76$, $\mathrm{p}<.01]$ and more quickly when given the position cue before the letter cue $[F(1,24)=9.30, p<.01]$. Again the interaction was not significant $[F(1,24)=1.33$, $p>.05]$, and all six pairwise comparisons reached at least the .05 level of significance.

The number of omissions (no response within $30 \mathrm{sec}$ ) and the number of errors were also different in each of the four conditions. When the first letter was provided, response omissions occurred $1.3 \%$ of the time; when the third letter was provided, omissions occurred $11.67 \%$ of the time. These percentages are significantly different from each other $\left[x^{2}(1)=24.67, p<.01\right]$. The error rates follow a similar pattern. When provided with the first letter, only $1.67 \%$ of the responses were errors; when the third letter was provided, errors occurred $7.33 \%$ of the time. These percentages are also significantly different $\left[\chi^{2}(1)=16.71, p<.01\right]$. A more detailed examination of the 22 errors made when the third letter was provided reveals that 17 of them contained the given letter, but in the wrong position. For example, two Ss responded with "daisy" when asked for the name of a flower which had " $S$ " in the third position.

\section{Discussion}

The basic finding of the present research is that it is easier to produce a category member that starts with a particular letter than one in which the particular letter is in the third position. Furthermore, giving $S$ the position he is to be concerned with before giving him the letter facilitates retrieval over the reverse arrangement. Thus, naming an "animal-1-D" is faster than naming an "animal-D-1." These results enable us to reject one explanation which is contradicted by the data and to tentatively propose a two-strategy model which does seem quite plausible.

Consider the possible strategy that, when $\mathrm{S}$ gets a letter first, he thinks of a category member beginning with that letter during the 0.5 -sec interval. When the position cue arrives, he is in luck if it is a " 1 ," for he then simply outputs his response. However, when the position cue " 1 " comes before the letter, $S$ must first find his response and then output it. The latter case should take longer, but it does not. Thus, it appears that $S$ does not use the interval between letter and number to think of a category member beginning with the letter.

As a framework for discussing the present results, we would like to outline a two-strategy model for retrieval. According to this model, the process of finding a category member that starts with a particular letter is different from the case in which the given letter is to be in the third position. In both cases, the first step of the processes involves finding the area of memory which corresponds to the given category (or "entering the category"). Evidence for this assumption can be found elsewhere (Freedman \& Loftus, 1971; Loftus \& Freedman, 1972). The next step is assumed to involve a search within the category for an appropriate category member, and it is at this stage that we assume two 
strategies are involved. Evidence for the existence of two strategies comes directly from the present data: When the position cue comes before rather than after the letter cue, Ss are approximately $500-700 \mathrm{msec}$ faster. What do Ss do with the position cue for $500-700$ msec? We propose that they use this information to decide which within-category search strategy they will use.

Consider first the strategy Ss use when finding a category member with a particular letter in its third position. After $S$ enters the appropriate category, it seems likely that he next goes through a preliminary elimination process, which consists of a quick check to see if the letter is in the word at all, and then a more detailed check to see if the letter is in the third position. The relatively high error rate for this case and the fact that the majority of the errors do contain the letter somewhere in the word suggest that the process sometimes terminates prematurely after the quick check. It may be that $\mathrm{S}$ feels he can respond without making sure that the letter is in the proper position. Given that $S$ is under some pressure to respond quickly, this strategy may not be so unreasonable.

A different situation obtains when $\mathbf{S}$ searches for a category member that begins with a given letter. In the present study, we cannot use the errors to give detailed information about the strategy-there are too few of them. In a large earlier study, however, in which Ss named category members that began with particular letters (Freedman \& Loftus, 1971), the few errors that occurred seemed to have the stimulus letter in the correct position and were errors because they were not true category members. For example, one $\mathrm{S}$ who was asked for a city beginning with " $M$ " said "Minnesota." Our current conception of retrieval in this case involves a quasi-parallel search through the category for a word beginning with the given letter. An error in which the given letter is present and in the correct position but the word itself is not a true category member could occur because $S$ has entered an inappropriate (although usually related) category.

\section{EXPERIMENT II}
Method
In order to get more information and to clarify the results of Experiment I, a second experiment was designed. This experiment was similar to Experiment $I$, except two major changes were incorporated. First, Ss saw only a number and a letter and were required to produce any word which had the given letter in the position designated by the given number. For example, Ss who were presented with the stimulus " $1-B$ " might say boat, bed, boring, or brat, among other possibilities. The reason we did not require $S s$ to produce category members is because we wanted more freedom in the use of letters, and we also wanted to be able to compare the same letter in different positions. The second procedural change was to use positions 1 , $2,3,4$, and "last."
Sixteen Ss received a total of 80 trials each. Each of 16 letters occurred five times, once in each of the five different positions. Half of the time the letter preceded the number cue (e.g., B-1); half the time it followed (e.g., 1-B). Half of the time the interval between the two cues was $0.5 \mathrm{sec}$; half the time it was $2.5 \mathrm{sec}$.

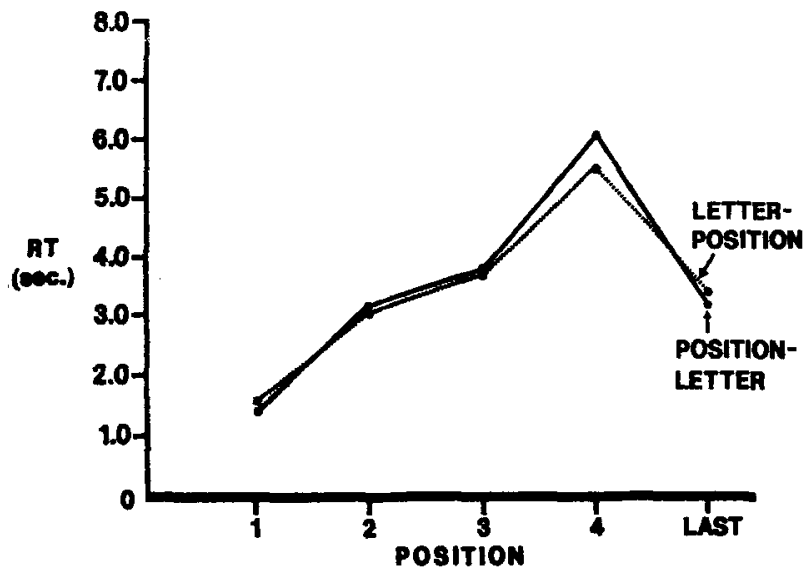

Fig. 2. Results of Experiment II. Mean RT as a function of position cue for position-letter and letter-position orders of presentation.

The apparatus and procedure were similar to those used in Experiment I.

\section{Results}

Only latencies for correct responses are included in the first two analyses. Responses were grouped into 20 different classifications depending on (1) position cue $(1,2,3,4$, or last), (2) order of position and letter cues (position first vs second), (3) interval $(0.5$ vs $2.5 \mathrm{sec})$. Median latencies were obtained for each S's responses in each of the 20 classifications. A three-way analysis of variance with repeated measures revealed the following significant effects. Position had a significant effect $[F(4,60)=31.44, p<.01]$. A Newman-Keuls procedure showed that Positions 1 and 4 were different from all others, while Positions 2, 3, and last were not different $(p<.05)$. No other main effects or interactions were significant $(F<1)$. Figure 2 presents mean RT as a function of position for both position-first and position-second methods of presentation. These means were obtained by averaging medians for individual Ss.

A similar analysis was done by letters rather than by Ss. Median latencies were obtained for the responses to each letter in each of the 20 stimulus classifications. A three-way analysis of variance indicated that position had a significant effect $[F(4,60)=16.55, p<.01]$, while no other effects were significant.

The error pattern was similar to that found in Experiment I. There were fewer than $2 \%$ errors when the first letter of the word was provided; however, there were $8 \%$ errors when the letter had to be placed in some other position. Furthermore, $81 \%$ of these latter errors contained the letter somewhere in the word, but in the wrong position.

\section{Discussion}

The order of the position and letter cues influenced RT in Experiment I but not in Experiment II. There were two procedural differences in the two experiments. In Experiment I, an $S$ had to find an item whose meaning was restricted (e.g., it had to be an animal); in 


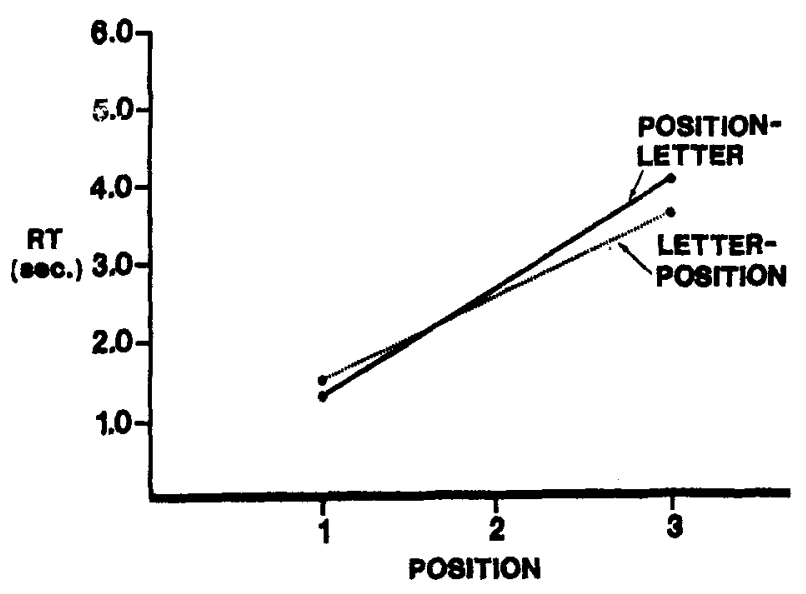

Fig. 3. Results of Experiment III. Mean RT as a function of position for position-letter and letter-position orders of presentation.

Experiment II, an S searched for an item which had to satisfy only orthographic constraints (e.g., a word which began with $Z$ ). In the first experiment, two positions were used throughout (Positions 1 and 3 ), while in the second experiment, five different positions were used.

There are two possible reasons why the dual strategy model suggested by the effect of order in Experiment I may not be operative in Experiment II. (1) The model might not be appropriate to situations where item meaning is unrestricted (i.e., finding an item whose meaning is restricted may involve a different process from finding an item whose meaning is unrestricted. (2) The model might not be appropriate in cases where many positions are used. It may be impractical to have five different strategies, one for each position, so perhaps only one strategy is used. To settle this issue, Experiment III was designed.

\section{EXPERIMENT III}

\begin{abstract}
Method
Twelve Ss saw 40 stimuli, each consisting of a number and a letter, and they were required to produce any word which had the given letter in the position designated by the given number. Twenty different letters were used, and each appeared once with the position cue " 1 " and once with " 3 ." Thus, the experiment was like Experiment $I$ in that only two positions were used throughout: 1 and 3 .

Half of the time the letter preceded the number cue (e.g., B-1) by $.5 \mathrm{sec}$; half of the time it followed it (e.g., 1-B). Other details about the apparatus and procedure were similar to Experiments I and II.
\end{abstract}

Results

Only latencies for correct responses are included in the first two analyses. Responses were grouped into four classifications depending on (1) the position cue (1 or 3$)$ and (2) the order of the position and letter cues (position first vs second). Median latencies were obtained for each S's responses in each of the 20 classifications. For each classification, group mean latencies were obtained by averaging medians for individual Ss, and these are plotted in Fig. 3. A two-way analysis of variance with repeated measures indicated that position had a significant effect $[F(1,11)=78.91$, $p<.01]$; however, order did not $(F<1)$. The interaction was not significant $[\mathrm{F}(1,11)=1.41$, $\mathrm{p}>.10]$.

A similar analysis done by letters rather than by Ss revealed that position was significant $[F(1,19)=78.91$, $\mathrm{p}<.01]$, while neither order nor the interaction were $(\mathrm{p}>.10)$.

\section{GENERAL DISCUSSION}

In three experiments, Ss produced words from semantic memory that satisfied different constraints. In Experiment I, semantic constraints were imposed, while in Experiments II and III, no semantic constraints were imposed. The effect of order in one case and not in the other strongly suggests that producing a word that satisfies certain semantic constraints involves a different process from producing a word that satisfies only certain orthographic restrictions.

At present we still do not completely understand how these two types of items are produced from memory. Intuitively, it feels as if you "find" an appropriate word in the "semantic" experiment, whereas, in the "word" experiment, you essentially "create" one. One S, when naming a word which was restricted in its third letter consistently placed "RE" before the letter and created a word from the trigram so produced. Thus, " $3-S$ " led to "rest," "3-P" led to "repeat," etc. The "semantic" and "word" mechanisms probably have some aspects in common, but they certainly have some differences. Lacking the ability to provide additional details about the retrieval processes, we present one additional argument indicating that semantic and word retrieval are probably quite different. If you present an $\mathbf{S}$ with an adjective (such as SMALL) and ask him to write down as many items as he can that have the inherent quality of the adjective, you will find his output is organized with regard to semantic properties (Loftus, 1972). For example, when asked to name "small" things, one S said "fly, bee, ant, eye, ear, toe, finger, grape, cherry, strawberry." When given a letter stimulus, however, and asked to name words that begin with that letter, something very different happens: Output is not organized with regard to semantic properties. For example, when asked to produce words beginning with "G," one $S$ began by saying "glue, glib, glide, gloat, gleam ..." The organization when given a letter is characteristically orthographic or phonemic but not, in general, semantic.

Why should we care if word and category decisions are different? For years and years, philosophers have been pushing the distinction between the word (or name) for an object and the semantic aspects (meaning) of the object (Quine, 1960). While some psychologists have recognized the distinction (Meyer \& Ellis, 1970), 
others have ignored it. For example, Landauer and Freedman (1968) do not make a distinction between deciding if the string of letters "DOG" is a word and deciding if "DOG" is an animal. Even though differential results were obtained, data from these two types of decisions were simply "averaged together." If there is a real distinction and real differences in processing, we must be careful about using data from one kind of decision to make inferences about the other. Furthermore, our models of memory retrieval must take account of these differences.

\section{REFERENCES}

Battig, W. F. \& Montague, W. E. Category norms for verbal items in 56 categories: A replication and extension of the Connecticut category norms. Journal of Experimental Psychology, 1969, 80,3.

Freedman, J. L., \& Loftus, E. F. Retrieval of words from long-term memory. Journal of Verbal Learning \& Verbal Behavior, 1971, 10, 107-115.
Kirk, R. E. Experimental design: Procedures for the behavioral sciences. Belmont, Calif: Brooks/Cole, 1968.

Landauer, T. K., \& Freedman, J. L. Information retrieval from long-term memory: Category size and recognition time. Journal of Verbal Learning \& Verbal Behavior, 1968, 7, 291-295.

Loftus, E. F. Nouns, adjectives, and semantic memory. Journal of Experimental Psychology, 1972, 96, 213-215.

Loftus, E. F., \& Freedman, J. L. Effect of category-name frequency on the speed of naming an instance of the category. Journal of Verbal Learning \& Verbal Behavior, 1972, 11, 343-347.

Meyer, D. E., \& Ellis, G. B. Parallel processes in word recognition. Paper presented at the meeting of the Psychonomic Society, San Antonio, November 1970.

Quine, W. V. Word and object. Cambridge, Mass: MIT Press, 1960.

Tversky, A., \& Kahneman, D. Availability: A heuristic for judging frequency and probability. Oregon Research Institute Bulletin, 1972,11, No. 6 .

(Received for publication November 23, 1973; revision received January 2, 1974.) 